\section{Cerebellar peduncle abscess secondary to disseminated strangles in a six-week-old miniature foal}

\section{Brianne Henderson \\ Dunnville Veterinary Clinic, Dunnville, Ontario, Canada}

\begin{abstract}
During a strangles outbreak within a herd of minature horses, a six week old foal developed acute onset clinical signs of sepsis and neurological deficits. The foal was euthanized and submitted for post-mortem at the Animal Health Laboratories, Guelph Ontario. Gross postmortem examination noted severe bronchopneumonia, hypopyon of the right eye and a singular cerebellar peduncle abscess. Culture of the lungs and cerebellum produced a pure growth of Streptococcus equi ssp. equi. Streptococcus equi ssp. equi, the causative agent of equine strangles, produces an acute pyrexia, purulent lymphadenopathy of submandibular and retropharyngeal lymph nodes. Commonly, lymph node abscesses rupture and resolve without complication. Rarely, complications may include: dissemination of the bacteria with diffuse abscess formation, immune mediated disease (purpura haemorrhagica), rarely abscess formation within the central nervous system (CNS) can occur. These can be managed medically with appropriate antibiotics and drugs to reduce intra-cranial pressure, however surgical drainage and debulking of the abscess has been attempted successfully in a few cases.
\end{abstract}

\section{Herd history}

In the spring of 2011,8 horses were introduced to a herd of 20 miniature horses. Neither group of animals had been immunized with core vaccines and no quarantine protocol was carried out by the owner. Initial clinical signs of mucupurulent nasal discharge, swollen submandibular lymph nodes and pyrexia were displayed by the imported horses; approximately one month later, the original herd displayed similar signs of disease. One 24-hour-old foal died suddenly over night and two 4-6-week-old foals were euthanized when they became recumbent and showed clinical signs of sepsis.

Naso-pharyngeal swabs were submitted for culture, produced a growth of Streptococcus equi ssp., zooepidemicus. Repeat naso-pharyngeal and abscess swabs submitted for PCR were taken approximately 1 week later. These yielded a positive result for Streptococcus equi ssp. equi.

\section{Six-week-old foal presentation}

A 6-week-old foal born on the farm was seen as an emergency. Physical examination found the foal in lateral recumbency, unable to maintain a sternal position. Pupillary light reflex was absent and horizontal nystagmus was noted in both eyes. Hypopyon of the right eye was observed. The foal had a temperature of $38.4^{\circ} \mathrm{C}$ and a heart rate of $60 \mathrm{bpm}$. Mucous membranes were pink but tacky. The foal was struggling to breathe and crackles were auscultated throughout the right lung, while reduced lung sounds were auscultated on the left. No signs of colic were observed.

Prior to euthanasia blood samples were collected for hematology and biochemistry, the results of which showed a marked mature neutrophilia with evidence of mild-moderate toxic change. Increased serum haptoglobin and serum amyloid A were consistent with significant ongoing tissue inflammation/infection. Concurrent elevations in acute phase proteins and a mature neutrophilia suggested chronicity of disease.

Based on a poor prognosis for recovery, the foal was euthanized by intravenous injection of pentobarbital and submitted to the Animal Health Laboratory, Guelph Ontairo, for postmortem and histopathology.

\section{Post-mortem}

Gross post-mortem results found the trachea and bronchi to contain viscous purulent exudates and a purulent bronchopneumonia of the antero-ventral half of the left caudal lobe. Multiple large abscesses (2-3 cm in diameter) were evident in all affected areas. The stomach was empty, while the small intestine was segmentally distended with green mucoid contents. Examination of the cranium revealed a $1 \mathrm{~cm}$ abscess of the cerebellar peduncular area.

Culture of the lung and brain abscesses produced a pure culture of Streptococcus equi ssp. equi.

\section{Histopathology}

Histopathology of the lung found a marked neutrophilic reaction within the lumens of some bronchioles. There was a marked generalized pulmonary congestion and oedema and erosion of the bronchiolar mucosa in affected lung lobes. Multiple abscesses, varying in size, were present. The lumen of these abscesses was filled with cellular debris, neutrophils and cocci. All of the abscesses were surrounded by macrophages, lymphocytes and plasma cells (Figure 1). Moderate lymphoid depletion and congestion of the red pulp was found within the spleen.
Correspondence: Dr. Brianne Henderson, Dunnville Veterinary Clinic, 110 Ramsey Drive Dunnville, Ontario N1A 1K8, Canada.

Tel. +1.905.7747642.

E-mail: morwen13@googlemail.com

Key words: strangles, abscess, foal, cerebellum, Streptococcus equi.

Acknowledgments: the author wishes to thank and acknowledge Dr. Tony VanDreumel, Dr. Durda Slavic and Dr. Murray Hazlett of the Animal Health Laboratory, Guelph Ontario, and Dr. Tom McQueen and Dr. Stan Alkemade for all of their work and contributions to the preparation of this case study.

The author is also very grateful to Kathy Zurbrigg of OMAFRA and the AHL Surveillance Project for providing an irreplaceable resource for gathering diagnostic information over the last two years.

Conflict of interest: the authors report no conflicts of interest.

Received for publication: 13 September 2011. Accepted for publication: 10 October 2011.

This work is licensed under a Creative Commons Attribution NonCommercial 3.0 License (CC BYNC 3.0).

(C) Copyright B. Henderson, 2011

Licensee PAGEPress, Italy

Veterinary Science Development 2011; 1:e12

doi:10.4081/vsd.2011.e12

The large abscess of the cerebellar peduncle area was examined closely. Marked peri-vascular cuffing primarily by neutrophils was evident. Hypertrophy of endothelial cells, leukocytosis and fibrin thrombi were also present in some capillaries (Figure 2).

\section{Discussion}

The causative agent of equine strangles is Streptococcus equi ssp. equi, which causes an acute pyrexia and purulent lymphadenopathy of the submandibular and retropharyngeal lymph nodes. ${ }^{1-3}$ Streptococcus equi ssp. equi is a highly host adapted Gram-positive, betahemolytic coccus bacteria that is transmitted via the oro-nasal route in nasal secretions or via fomites. ${ }^{1}$ The bacterium gains entry via the cryptal lymphoepithelium cells of the nasopharyngeal tonsil, which are continuous with the lymphoid tissue directly beneath. ${ }^{3}$ Small numbers of bacteria are able to gain access to the tonsillar tissue, at which point they slowly replicate until they disseminate throughout the tonsils and regional lymph nodes. ${ }^{3}$ Typically, abscesses rupture, drain and resolve 
with few complications. However, complications that may occur include: i) abscessation of retropharyngeal lymph nodes that can lead to guttural pouch empyema, chondroid formation and chronic shedding of the bacterium; ${ }^{1}$ ii) immune mediated conditions such as purpura haemorrhagica, myopathies and myocarditis; ${ }^{4}$ iii) dissemination of bacteria leading to a condition colloquially known as Bastard Strangles, which may involve abscessation sites in the lung, mesentery, liver, spleen, kidneys and brain. ${ }^{5}$ While there have been reports of metastatic abscesses in the central nervous system (CNS), these are considered to be a rare sequelae of this disease.

Factors involved in the likelihood of metastatic spread include: i) infection dose; ii) variation in strain virulence; and iii) variations in host susceptibility. The immunological naivete of this foal likely played the largest role in the rapid dissemination of bacteria. Mechanisms of dissemination include lymphatic migration, haematogenous spread ${ }^{2}$ or per continuitatem; when infected structures are in close or connected proximity to other vulnerable structures ${ }^{5}$ (i.e. retropharyngeal

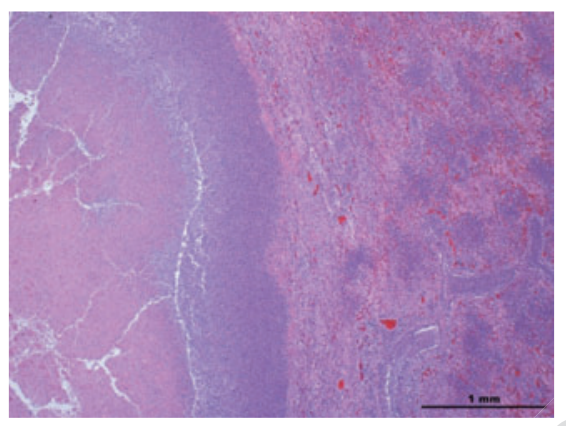

Figure 1. Severe neutrophil and macrophage infiltration of the lung parenchyma consistent with abscess formation.

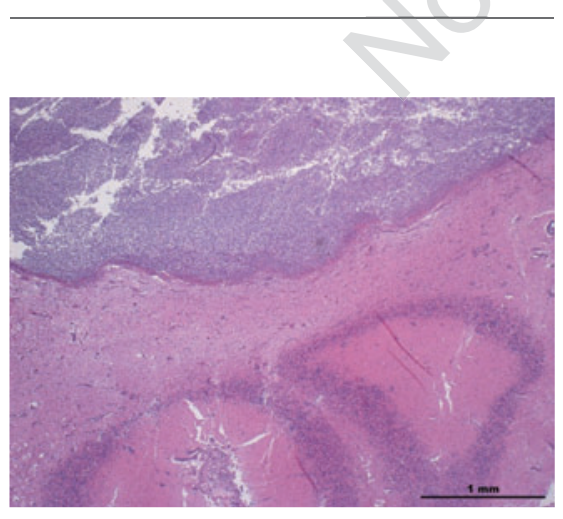

Figure 2. Cerebellar peduncle abscess. lymph node spread to the guttoral pouches or spread via the cranial nerves). Haematogenous spread or local extension is the most likely mechanism of dissemination in this case, as lymphatic migration does not reach the central nervous system. ${ }^{5}$

In contrast to human patients, bacterial meningitis/abscessation in horses and ruminants is a typical sequelae to systemic disease. Alternative theories of local extension have been proposed, whereby upper respiratory infections, dental disease, periocular lesions and traumatic head injuries could offer a primary septic foci from which local extension to the CNS could occur.5,6

The presence of multiple CNS abscesses and purulent meningitis alongside pneumonia and abdominal abscess formation suggest hematogenous spread. 5 Furthermore, hypopyon is a confirmed indicator of haematogenous spread. ${ }^{7}$ In this case, a single cerebellar peduncle abscess was found alongside diffuse, severe bronchopneumonia and hypopyon of the right eye. Based on the biochemical/ haematological markers of severe inflammation and advanced sepsis, haematogenous spread is the most likely pathway. However, the close anatomical association between the cerebellar peduncle and optic chiasm prevents local extension from being excluded as a possible pathogenesis of the single cerebellar peduncle abscess.

Medical management of bacterial meningioencephalitis consists of concurrent administration of corticosteroids, osmotic agents and antibiotics $^{8}$ to restore blood-brain barrier permeability, reduce intra-cranial pressure and control the inciting infection. A combination of anti-inflammatories (dexamethasone), mannitol and specifically bactericidal antibiotics are recommended. Dimethylsulfoxide (DMSO) has been used to reduce intracranial pressure (ICP); however, a severe rebound increase in ICP has been noted. 8

In addition, mannitol has a greater efficacy when used in properly hydrated animals. A surgical approach to intracranial abscesses has been described in the literature previously. ${ }^{8}$ Surgical drainage and debulking has been attempted to relieve intracranial pressure and remove the infective foci, with minimal success. Prognosis can be increased through the use of CT or MRI to map the lesion prior to surgery. 8,9 The primary goals of surgical debridement should be: i) clinical diagnosis/biopsy; ii) intracranial mass debulking, resection and decompression of neural tissue. ${ }^{8}$

Metastatic spread of Streptococcus equi ssp. equi to the CNS is a rare occurrence. Route of entry can be haematogenous, local extension or invasion via the cranial nerves. ${ }^{6}$ Multi-focal abscess formation is a strong indicator of haematogenous spread. Local extension of infectious foci has been highlighted as a possible route, specifically when discussing the closely related structures of the equine head. ${ }^{6}$ In this case, the singular cerebellar peduncule abscess could have formed from either haematogenous spread or local extension via the optic nerve.

The significant lesion identified in this foal highlights the need for meticulous preventative medicine and biosecurity protocols when introducing new horses to a herd.

\section{References}

1. Sweeney CR, Whitlock RH, Meris DA. Complications associated with Streptococcus equi infection on a horse farm. $\mathrm{J}$ Am Vet Med Assoc 1987;191:1446-8.

2. Timoney JF. Strangles. Vet Clin North Am 993;9:365-74.

3. Timoney JF, Kumar P. Early pathogenesis of equine Streptococcus equi infection (strangles). Equine Vet J 2008;40:637-42.

4. Whelchel DD, Chaffin MK. 2009. Sequelae and complications of Streptococcus equi subspecies equi infections in the horse. Equine Vet Educ 2009;21:135-41.

5. Spoormakers TJP, Ensink JM, Goehring LS, et al. Brain abscesses as a metastatic manifestation of strangles: symptomatology and the use of magnetic resonance imaging as a diagnostic aid. Equine Vet $\mathrm{J}$ 2003;35:146-51.

6. Smith JJ, Provost PJ, Paradis MR. Bacterial meningitis and brain abscesses secondary to infectious disease processes involving the head in horses: seven cases (1980-2001). J Am Vet Med Assoc 2004; 224:739-42.

7. Baggot JD. Drug therapy in the neonatal foal. Vet Clin North Am Equine Pract 1994;10:87-107.

8. Cornelisse JC, Schott HC, Lowrie CT, Rosenstein DS. 2001. Successful Treatment of Intracranial abscesses in 2 horses. J Vet Inter Med 2001;15:494-500.

9. Janicek JC, Kramer J, Coates JR, et al. Intracranial abscess caused by Rhodococcus equi infection in a foal. J Am Vet Med Assoc 2006;228:251-3. 